\title{
Photolithographically Manufactured Acrylate Polymer Multimode Optical Waveguide Loss Design Rules
}

\author{
Kai Wang, David R. Selviah, Ioannis Papakonstantinou', Guoyu Yu², Hadi Baghsiahi and F. Aníbal Fernández \\ Department of Electronic and Electrical Engineering, UCL, Torrington Place, London WC1E 7JE UK \\ k.wang@ee.ucl.ac.uk, d.selviah@ee.ucl.ac.uk, h.baghsiahi@ee.ucl.ac.uk, a.fernandez@ee.ucl.ac.uk \\ ${ }^{1}$ Sharp Laboratories of Europe Ltd (formally at UCL), Edmund Halley Road, Oxford Science Park, OX4 4GB \\ ${ }^{2}$ Ultra Precision Surfaces, UCL, Optic Technium, Fford William Morgan, St Asaph Business Park, LL17 0JD
}

\begin{abstract}
This paper describes how design rules are established for photolithographically manufactured acrylate polymer optical multimode waveguide components by optical experimental measurements made on the manufactured waveguide component. The loss of individual waveguide components, such as straight sections, $90^{\circ}$ bends, crossings, tapers and tapered bends must be known so that the combined loss of a cascade of such elements can be found to determine whether the interconnection's optical power budget is sufficient to achieve a good bit error rate. However, the loss depends on several factors: the materials: the polymer used for the core and for the cladding, the fabrication technique: e.g. the photolithographic procedure and the precise temperature baking regime used, and the measurement technique: the optical source lateral size and angular divergence and precise position relative to the entrance of the waveguide, the output detector lateral size, its angular acceptance angle (if any) and its precise position relative to the exit of the waveguide. The experiments reported on photolithographically manufactured acrylate polymer multimode waveguide were performed at room temperature. A new technique for measure the transmitted power at waveguide crossings is reported for the first time.
\end{abstract}

\section{Introduction}

Optical interconnections are being investigated for short distance, high speed, data communication applications on printed circuit boards (PCB) to replace copper tracks which suffer severe cross-talk as data rates rise above $10 \mathrm{~Gb} / \mathrm{s}$, as well as increased loss and increased cost. Optical beams can pass through one another in free space without any cross-talk so offer an attractive alternative provided the cost of the optical interconnections can be minimized. The lowest cost approach is to form polymer waveguides with a higher refractive index polymer core surrounded by a lower refractive index polymer cladding, [1-3] fabricated on multi-layer optical PCBs (OPCBs) which has the advantage that the PCB copper tracks can carry power and low data rate control signals to line cards.

We designed test masks to measure key parameters such as loss. The waveguide structures included straight waveguides of various widths, novel tapered bends [4], crossings between two waveguides at a range of angles and bends of a range of radii. Once the optical loss has been measured for each waveguide component, waveguide design rules can be established for different waveguide manufacturing techniques, e.g. photolithography, direct laser-writing, laser ablation, embossing, extrusion and printing and incorporated into commercial automatic design rule checker and constraint manager layout software for PCBs so that PCB designers can easily include optical connection layers without detailed knowledge of the optics involved. The optical board layout can then be optimised to minimise the waveguide loss and optical cross-talk. Design rules are being embedded in future generation of E-CAD for integrated design and layout software for electronic and photonic interconnect.

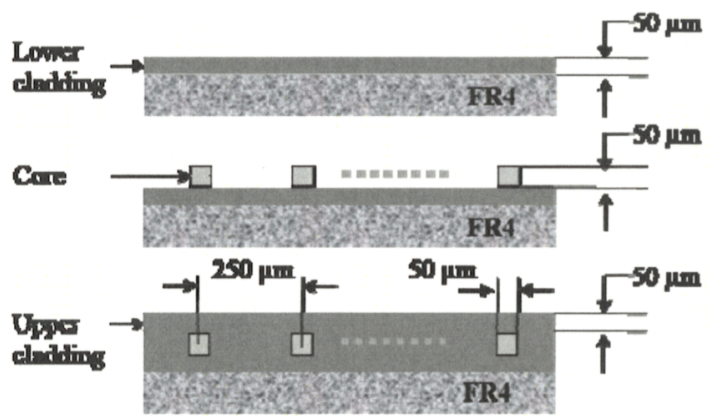

Fig. 1 Photolithographically manufactured OPCB, $50 \mu \mathrm{m} \times 50 \mu \mathrm{m}$ core

Waveguides were photolithographically fabricated from Truemode ${ }^{\circledR}$ polymer [5] on an FR4 PCB. The refractive index of the core was 1.5560 and the cladding was 1.5264 giving an NA=0.302. The photolithographic process was optimised for $50 \mu \mathrm{m}$ thick waveguides. After fabrication the thicknesses of the lower and upper claddings were $50 \mu \mathrm{m}$ respectively, the waveguide core was $50 \mu \mathrm{m}$ thick unless otherwise stated, and the pitch between two adjacent waveguides was $250 \mu \mathrm{m}$ (see Fig. 1). The Truemode ${ }^{\circledR}$ polymer has been measured to have a propagation loss of $0.03-0.06 \mathrm{~dB} / \mathrm{cm}$ [6] at $850 \mathrm{~nm}$, which is ideal for use with $10 \mathrm{~Gb} / \mathrm{s}$ VCSELs. Masks are made by e-beam lithography to give the highest resolution so that the propagation loss is dominated by waveguide side wall roughness caused by fabrication rather than mask resolution error.

\section{Measurement technique}

The measurement results depend strongly on the measurement technique, i.e. the choice of optical source: VCSEL direct coupling or launched via an optical fibre, the condition of the entrance of the waveguide, the type of output detector and more 
importantly the relative positions of all of those components. We used light from an $845 \mathrm{~nm}$ VCSEL (ULM photonics, ULM850-10-TT-C010104U) launched into a standard 50/125 $\mu \mathrm{m}$ step index MM fibre with $N A_{\text {fibre }}: 0.2<$ waveguide $N A_{\text {wg }}: 0.302$. The fibre was wound sufficient times around a steel post to couple the modes in order to fill the solid angle of the numerical aperture with a large number of transverse modes. The fibre was aligned and butt-coupled to one of the waveguides. Light from the waveguide output was spatially filtered by a $150 \mu \mathrm{m}$ diameter circular pinhole for the crossing experiment reported to exclude much of the light travelling through the cladding. For the crosstalk, bend and tapered bend experiments a $70 \mu \mathrm{m}$ pinhole was chosen to simulate the aperture of the photodetector (PD) chosen for use in the final practical system demonstrator [7]. A large area integrating sphere photodetector was placed after the output pinhole to measure the integrated output optical power so avoiding inconsistencies due to laser speckle and spatial variation of efficiency across the photodiode detector. Index matching fluid ( $n=1.4911$ at $845 \mathrm{~nm}$ ) was applied to both MM fibre - waveguide and waveguide - pinhole interfaces to reduce coupling loss. Both the input fibre and PD were mounted on high precision sub-micron motorized translation stages for accurate alignment and step adjusted to maximise the light through the waveguide.

\section{Cross-talk between straight waveguides}

Cross-talk between neighbouring waveguides is one of the critical factors which could significantly affect the performance of a high-bit-rate link. A sample containing an array of straight waveguides with similar widths was used to characterize cross-talk. Each waveguide was designed to have an approximately square cross section of $70 \mu \mathrm{m}$ to maximize the misalignment possible while minimizing the coupling loss for the VCSEL and PD diameters chosen. The array of 12 straight waveguides were on a pitch of $250 \mu \mathrm{m}$ and were $10 \mathrm{~cm}$ long to fit onto a 6 " diameter circular FR4 wafer substrate of $800 \mu \mathrm{m}$ thickness coated in a 17 $\mu \mathrm{m}$ copper layer on the opposite side of the waveguides. An $850 \mathrm{~nm}$ VCSEL imaged by a GRIN-lens [7] was directly coupled to a waveguide at $y=0, z=0$ (named $0^{\text {th }}$ waveguide in Fig. 2).

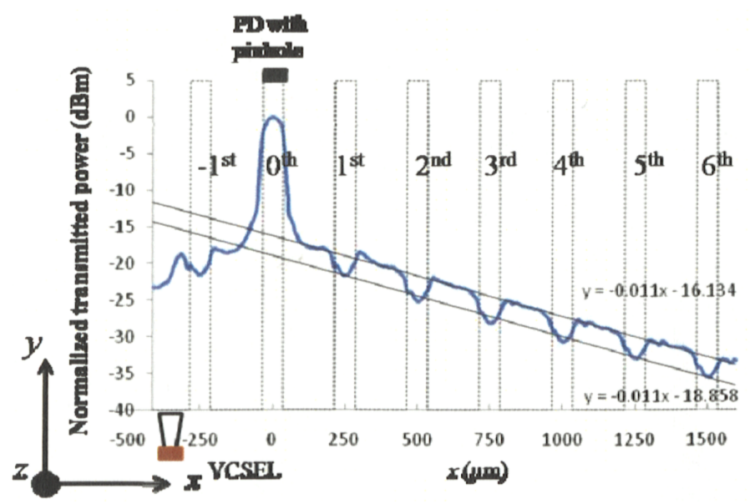

Fig. 2 Cross talk in straight waveguides
Once the positions of VCSEL and PD were optimized for receiving maximum transmitted power at the $0^{\text {th }}$ waveguide, the VCSEL laterally scanned across the waveguides from $-300 \mu \mathrm{m}$ to $1600 \mu \mathrm{m}$ in $1 \mu \mathrm{m}$ step increments along the $x$ axis, while the power at the end of the $0^{\text {th }}$ waveguide was monitored at every step. Misaligned components both contribute to increased insertion loss and increased cross-talk. For as long as the VCSEL emits between the boundaries of the $0^{\text {th }}$ waveguide the recorded power corresponded to the power coupled into this waveguide as a function of the lateral misalignment, $x$. When the VCSEL emits into the cladding, the power drops almost linearly at a rate of $0.011 \mathrm{dBm} / \mu \mathrm{m}$ (see Eq. (1)), as its distance increases from the $0^{\text {th }}$ waveguide centre. However, when the VCSEL emits into the core area of the waveguides next to the $0^{\text {th }}$ waveguide, the power detected corresponded to cross-talk originating from other VCSELs of the same array that would be coupled to these waveguides in a system.

$$
y=-0.011 x-16.134
$$

A sudden power drop is observed in the neighbouring waveguides due to power trapped and confined within the waveguide cores. The lowest cross-talk levels were achieved when perfect alignment occurs between the VCSELs and the waveguides. As the VCSELs move away from the centre of the other waveguides, while still remaining in the core area, the received power increases indicating worsening of cross-talk. In the case of perfect alignment, two neighbouring waveguide power valley minima have a linear relationship at the rate of $0.011 \mathrm{dBm} / \mu \mathrm{m}$ (Eq. (2)).

$$
y=-0.011 x-18.858
$$

\section{Waveguide crossing design rules}

Optical systems allow one data channel to pass through another one in the same layer, which is not the case for copper tracks. However, a proportion of the input light is lost at each crossing channel depending on the crossing angle. A schematic diagram of a waveguide with $90^{\circ}$ crossings is shown in Fig. 3.



Fig. 3 Schematic diagram of $90^{\circ}$ waveguide crossings 
Curved waveguide I has a straight input waveguide $l_{i n}$, followed by a circular arc section at $90^{\circ}$. Six straight waveguides cross through the central straight section $l_{c}$ at $90^{\circ}$. Another identical $90^{\circ}$ circular arc section, but bent in the opposite direction, guides the channel to the final output straight waveguide $l_{o u t}$.

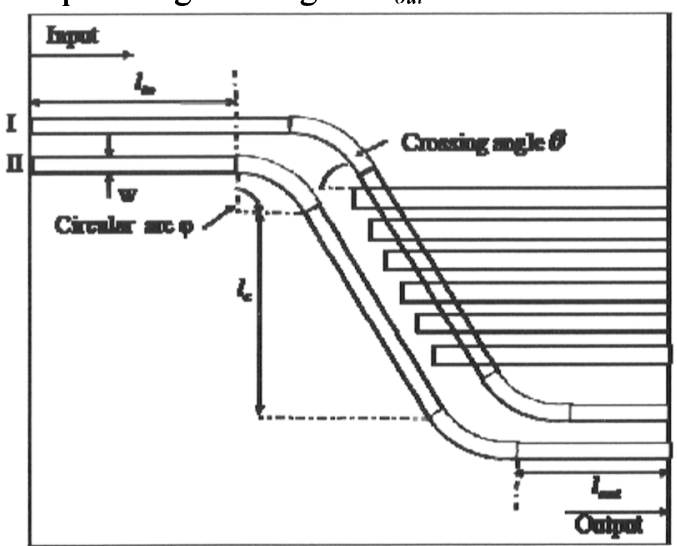

Fig. 4 Schematic diagram of waveguide crossings at an arbitrary angle, $\theta$

Fig. 4 shows a straight input waveguide $l_{i n}$, followed by a circular arc section of $\theta$ which controls the orientation of the central straight waveguide $l_{c}$ giving a designed crossing angle, $\theta$. The crossing angles, $\theta$ were $10^{\circ}, 15^{\circ}, 20^{\circ}, 25^{\circ}$, and from $30^{\circ}$ to $90^{\circ}$ in an increments of $10^{\circ}$ giving 11 different crossing angles. The curved waveguide design provides a filter effect to exclude much of the light travelling through the cladding which from the original input direction [10] might be captured by an in line integrating sphere photodetector.

The calibrated transmitted power at each crossing as a function of the crossing angle is shown in Fig. 5. The vertical axis is defined as the power measured at the end of waveguide I containing 6 crossings minus the power measured at the end of the waveguide II in the same group (Fig. 3) divided by 6 . Since the radius, angle of the arc and the total length of straight sections are the same in both curved waveguide I and II, this calibration step removes all the additional losses.

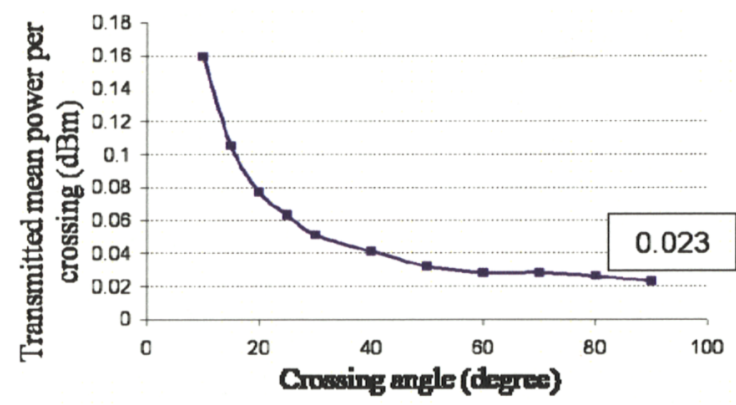

Fig. 5 Transmitted mean power per crossing as a function of crossing angles

We used the combination of crossings and the curved waveguide to measure loss per crossing and achieved a consistent result with that of other workers $[8,9]$ for the $90^{\circ}$ case. We measured each waveguide 50 times for each designed crossing angle. The arithmetic mean for each point is shown in Fig. 5. The transmitted mean power of $0.023 \mathrm{~dB}$ per crossing was achieved at a $90^{\circ}$ crossing which means that the output power dropped by $0.5 \%$ at each $90^{\circ}$ crossing. Fig. 5 can be used as a design curve to establish the loss for the link power budget calculation.

\section{Design rules for bends}

The modes in waveguide bends tend to have their energy shifted towards the outside of the bend and are inherently lossy. More importantly, bends are usually attached to other, for example, straight waveguides resulting in a modal mismatch and additional loss at the transition point. The different types of loss radiate in different directions from the waveguide [10]. The relative strengths of the various types of loss must be found so that other waveguides on the backplane can be arranged to reduce crosstalk and optimise the layout of an optical data link. Therefore, carefully arranged experiments must be designed to separate the total bend loss from the input and output coupling loss. Loss vs. radii curves allow engineers to choose the appropriate bend radius and to layout the waveguides to minimize crosstalk [10].

Fig. 6 shows a schematic diagram of one set of nested bends containing waveguide tapered bends, bends and straight tapers plus bends used in the experiments. The mask pattern was designed to have $90^{\circ}$ circular arc waveguide bends and tapered bends aligned to $l_{\text {in }}=11.5 \mathrm{~mm}$ input straight sections and $l_{\text {out }}=24.5$ $\mathrm{mm}$ output straight sections. Three sets of waveguides with the same thickness, $50 \mu \mathrm{m}$, but different widths, $w_{\text {in }}$ $=50 \mu \mathrm{m}, 75 \mu \mathrm{m}$, and $100 \mu \mathrm{m}$ were fabricated on the same 6" circular FR4 PCB wafer.

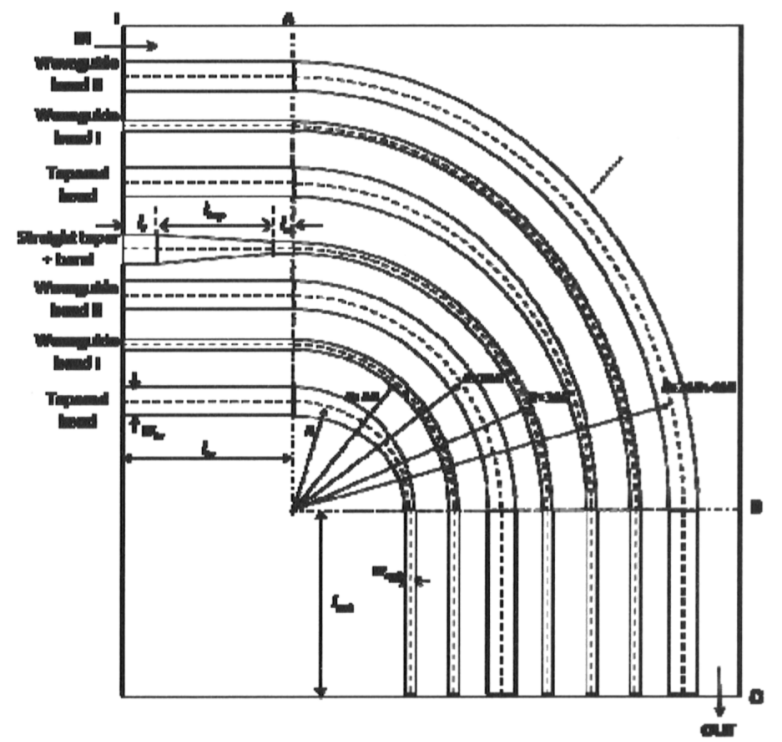

Fig. 6. Schematic diagram of one part of nested waveguide bends.

The waveguide with the smallest radius $R$, is the tapered waveguide bend. The output widths of the tapered bends were $w_{\text {out }}=10 \mu \mathrm{m}, 20 \mu \mathrm{m}, 25 \mu \mathrm{m}, 30 \mu \mathrm{m}$ and $40 \mu \mathrm{m}$ giving corresponding taper ratios $T R=0.2$, $0.4,0.5,0.6$ and $0.8,\left(T R=w_{\text {out }} / w_{\text {in }}\right)$. The adjacent component with radius $R+\Delta R$, is an input straight bend - output straight waveguide cascade (waveguide bend I). All three waveguide sections in this case have the same width $w_{\text {out }}$, which can also be considered to be a special case of a tapered bend with $T R=1$. The 
separation between any two successive waveguide optic axes is $\Delta R=250 \mu \mathrm{m}$. The next component with radius $R+2 \Delta R$, is a second waveguide bend, where all of the waveguide sections have width $w_{i n}$, (waveguide bend II). The fourth component $(R+3 \Delta R)$, is a combination of an input straight - straight tapered - output straight $l_{0}$ bend - output straight $l_{\text {out }}$, waveguides (we refer to this component as the straight taper plus bend). The input straight waveguide has length $l_{i}=1 \mathrm{~mm}$ and width $w_{i n}$. The straight tapered waveguide is a linear taper from $w_{i n}$ to $w_{\text {out }}$ with length $l_{\text {tap }}=10 \mathrm{~mm}$ with the output straight waveguide $l_{o}=0.5 \mathrm{~mm}$ attached to the taper. The curved section is a $90^{\circ}$ bend of radius $R+3 \Delta R$, and width $w_{\text {out }}$ followed by the output straight section $l_{\text {out }}$ at the same width $w_{\text {out }}$. This component was designed to compare the performance between straight tapers and tapered bends.

Each bend structure re-appears once at every $4 \Delta R$ with increasing taper ratio. The first group had $T R=0.2$ and started from $R=5 \mathrm{~mm}$, followed by a second concentric group with $T R=0.4$ starting at $R=6 \mathrm{~mm}$, a third with $T R=0.5$ at $R=7 \mathrm{~mm}$ and so on until all 5 designed values of $T R$ had been used then the sequence repeats again until the last group starts $R=34 \mathrm{~mm}$. There were 6 waveguide groups for each value of $T R$ but having different radius of curvature, $R$. The waveguide bend II with a uniform width $w_{i n}$ starts from a minimum radius, $R=5.5 \mathrm{~mm}$ and increases in radius in increments of $4 \Delta R=1 \mathrm{~mm}$, to a maximum radius of $\mathrm{R}=34.5 \mathrm{~mm}$ giving a total number of waveguide bends $N=30$ in each set.

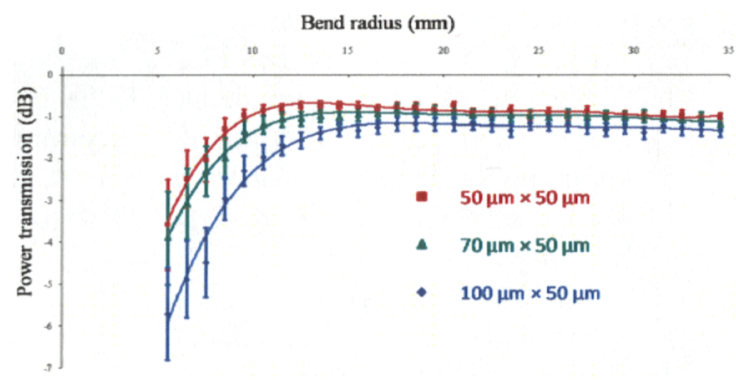

Fig. 7. Power transmission of waveguide bends for three widths $w$ $=50 \mu \mathrm{m}, 75 \mu \mathrm{m}$ and $100 \mu \mathrm{m}$

Results were calibrated using the measurement from a straight waveguide fabricated on the same sample. Normalized transmitted power as a function of the radius of curvature is shown in Fig. 7 for the three sets with different waveguide widths. Radiation and transition loss dominated when $R<20 \mathrm{~mm}$ [11-15] while propagation loss becomes more significant in larger radius bends due to longer lengths.

Table 1 Optimum radius for different waveguide widths

\begin{tabular}{c|c|c}
\hline Width $(\mu \mathrm{m})$ & $\begin{array}{c}\text { Optimum Radius } \\
(\mathrm{mm})\end{array}$ & $\begin{array}{c}\text { Minimum Loss } \\
(\mathrm{dB})\end{array}$ \\
\hline 50 & 13.5 & 0.74 \\
75 & 15.3 & 0.91 \\
100 & 17.7 & 1.18
\end{tabular}

The loss reaches a minimum at $R \sim 15 \mathrm{~mm}$ in all cases as shown in Table 1 . The wider waveguides have consistently higher insertion loss.

\section{Design rules for tapered bends}

Wide straight waveguides give large input misalignment tolerances for small aperture lasers, but their outputs might be larger than the active area of the photodetectors causing excess loss and modal noise. The high speed photodetector area is limited to minimize their capacitance and maximize their speed. A large input aperture waveguide is needed which tapers down in width to a narrow output aperture, but tapered waveguides can be lossy unless they taper down slowly to minimize coupling between propagating and radiating modes so they must be long. However, in OPCBs, waveguides often have to bend almost immediately after the connector to avoid other connectors or obstacles and, therefore, there is not much space available for long tapers. A solution, which can result in a more compact device, is to combine a taper and a bend in a single component, the tapered bend [16]. We measured tapered bends [4] using the same sample in Fig. 6 .

To investigate the effect of source misalignment the MM fibre of the previous experiments was replaced with a single mode (SM) fibre and scanned along the lateral direction $x$. Fig. 9 compares one scan for a tapered bend with TR $=0.4$ and $w_{\text {out }}=20 \mu \mathrm{m}$ with one for a waveguide bend with $w_{i n}=w_{\text {out }}=20 \mu \mathrm{m}$. Although they both have the same width at their output, the tapered bend maintains high coupling efficiency for a larger range of fibre misalignment than the waveguide bend but at an increased loss penalty.

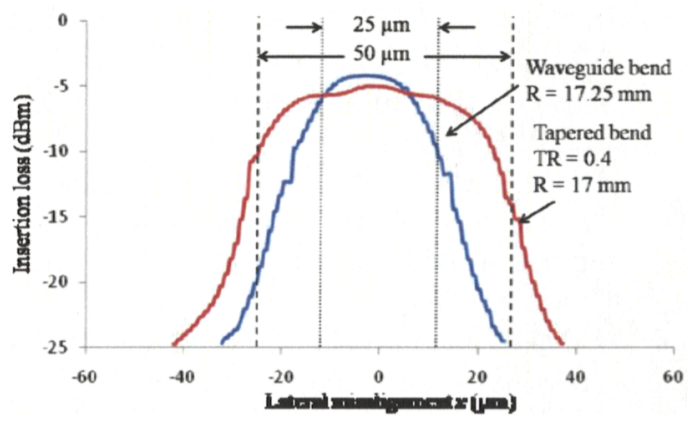

Fig. 8. Insertion loss for a waveguide tapered bend and a bend

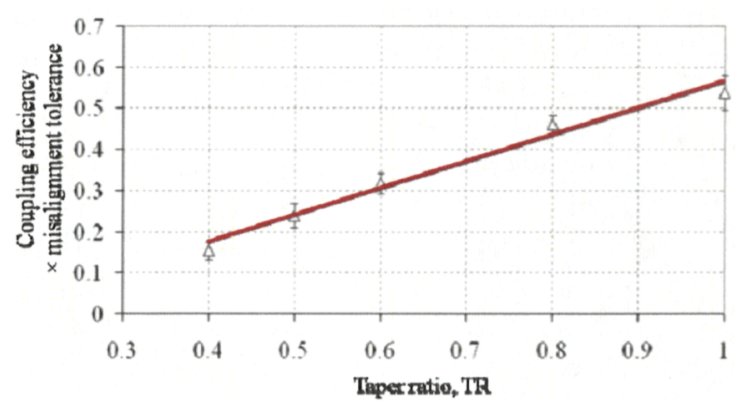

Fig. 9. Coupling efficiency times misalignment tolerance as a function of $T R$.

Coupling efficiency times misalignment tolerance of 
tapered waveguide bends shows a linear relationship with $T R$ in Fig. 9 which suggests that $T R$ is almost unrelated to the radius of curvature. The effect of $R$ on loss can be found from design curves for waveguide bends [10]. The product of insertion efficiency and misalignment tolerance for tapered bends is $0.650 T R$ 0.09 .

\section{Conclusions}

This paper characterised photolithographically manufactured acrylate polymer multimode waveguide by measuring the optical loss of key waveguide components. Design rules derived from the experimental measurement to assist optical system designers to optimise OPCB layout.

\section{Acknowledgments}

The authors thank EPSRC via IeMRC for funding and Dave Milward, for managing the project, Ken Hopkins, and Richard Pitwon, Xyratex Technology Ltd, for helpful discussions. Navin Suyal and Habib Rehman, Exxelis for preparation of the Truemode ${ }^{\circledR}$ polymer and photolithographic fabrication of the waveguides, Gary Hinde, Cadence for technical support.

\section{Reference}

1. Griese, E., "A high-performance hybrid electricaloptical interconnection technology for high-speed electronic systems," IEEE Transactions on Advanced Packaging, Vol. 24, No. 3, 375-383, 2001.

2. Mederer, F., Jager, R., Unold, H. J., Michalzik, R., Ebeling, K. J., Lehmacher, S., Neyer, A., and Griese, E., "3-Gb/s data transmission with GaAsVCSELs over PCB integrated polymer waveguides," IEEE Photonics Technology Letters, Vol. 13, No. 9, 1032-1034, 2001.

3. Chen, R. T., Lin, L., Choi, C., Liu, Y. J. J., Bihari, B., Wu, L., Tang, S. N., Wickman, R., Picor, B., Hibbs-Brenner, M. K., Bristow, J., and Liu, Y. S., "Fully embedded board-level guided-wave optoelectronic interconnects," Proceedings of the IEEE, Vol. 88, No. 6, 780-793, 2000.

4. Papakonstantinou, I., Selviah, D. R., and Wang, K., "Insertion Loss and Misalignment Tolerance in Multimode Tapered Waveguide Bends," Ieee Photonics Technology Letters, Vol. 20, No. 12 2008.

5. "Truemode ${ }^{\circledR}$ wetfilm core datasheet," data accessed at 2003.

http://www.exxelis.com/products/truemode.php

6. Beals, J., Bamiedakis, N., Penty, R. V., White, I. H., DeGroot, J. V., and Clapp, T. V., "Modal noise investigation in multimode polymer waveguides art. no. 67810Q," Passive Components and FiberBased Devices Iv, Pts 1 and 2, Vol. 6781, Q7810, 2007.

7. Papakonstantinou, I., Selviah, D. R., R.A.Pitwon, and Dave Milward, "Low cost, precision selfalignment technique for coupling laser and photodiode arrays to waveguide arrays," IEEE Transactions on Advanced Packaging, 2008.

8. Sakamoto, T., Tsuda, H., Hikita, M., Kagawa, T., Tateno, K., and Amano, C., "Optical interconnection using VCSELs and polymeric waveguide circuits," Journal of Lightwave Technology, Vol. 18, No. 11, 1487-1492, 2000.

9. Bona, G. L., Offrein, B. J., Bapst, U., Berger, C., Beyeler, R., Budd, R., Dangel, R., Dellmann, L., and Horst, F., "Characterization of parallel opticalinterconnect waveguides integrated on a printed circuit board," Micro-Optics, VCSELS, and Photonic Interconnects, Vol. 5453, 134-141, 2004.

10. Papakonstantinou, I., Wang, K., Selviah, D. R., and Ferandez, F. A., "Transition, radiation and propagation loss in polymer multimode waveguide bends," Optics Express, Vol. 15, No. 2, 669-679, 22-1-2007.

11. Musa, S., Borreman, A., Kok, A. A. M., and Diemeer, M. B. J., "Experimental study of bent multimode optical waveguides," Applied Optics, Vol. 43, No. 30, 5705-5707, 20-10-2004.

12. L.Dellmann, R.Dangel, R.Beyeler, Ch.Berger, F.Horst, B.J.Offrein, and G.L.Bona, "Polymer waveguides for high-speed optical interconnects," Proceedings of EOS Topical Meeting on Optics in Computing, 131-132, 2004.

13. Hikita, M., Tomaru, S., Enbutsu, K., Ooba, N., Yoshimura, R., Usui, M., Yoshida, T., and Imamura, S., "Polymeric optical waveguide films for short-distance optical interconnects," Ieee Journal of Selected Topics in Quantum Electronics, Vol. 5, No. 5, 1237-1242, 1999.

14. Snyder, A. W. and Love, J. D., "Reflection at A Curved Dielectric Interface - Electromagnetic Tunneling," IEEE Transactions on Microwave Theory and Techniques, Vol. MT23, No. 1, 134141, 1975.

15. Goyal, I. C., Gallawa, R. L., and Ghatak, A. K., "Bent Planar Wave-Guides and Whispering Gallery Modes - A New Method of Analysis," Journal of Lightwave Technology, Vol. 8, No. 5, 768-774, 1990.

16. Wu, M. L., Fan, P. L., and Lee, C. T., "Completely adiabatic S-shaped bent tapers in optical waveguides," IEEE Photonics Technology Letters, Vol. 9, No. 2, 212-214, 1997. 